\title{
Partially Hydrolyzed Whey Infant Formula: Literature Review on Effects on Growth and the Risk of Developing Atopic Dermatitis in Infants from the General Population
}

\author{
Julien Sauser ${ }^{\mathrm{a}}$ Sophie Nutten ${ }^{\mathrm{a}}$ Nanda de Groot ${ }^{\mathrm{b}}$ Sophie Pecquet ${ }^{\mathrm{b}}$ \\ Dagmar Simon $^{c}$ Hans-Uwe Simon ${ }^{d}$ Jonathan M. Spergel ${ }^{e}$ Sibylle Koletzko ${ }^{f}$ \\ Carine Blanchard ${ }^{\mathrm{a}}$

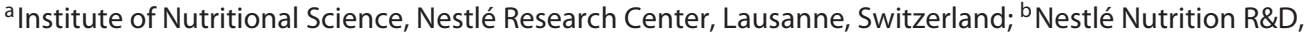 \\ Vevey, Switzerland; ' Department of Dermatology, Inselspital, Bern University Hospital, Bern, Switzerland; \\ ${ }^{d}$ Institute of Pharmacology, University of Bern, Bern, Switzerland; ' Division of Allergy and Immunology, \\ Department of Pediatrics, The Children's Hospital of Philadelphia, Perelman School of Medicine at University \\ of Pennsylvania, Philadelphia, PA, USA; ${ }^{\mathrm{f} D r}$. von Hauner Children's Hospital, Department of Pediatrics, \\ University Hospital, LMU Munich, Munich, Germany
}

\section{Keywords}

Atopic eczema · Formula $\cdot$ Growth $\cdot$ Infants $\cdot$ Nutrition

\begin{abstract}
Limited evidence is available regarding the effect of partially hydrolyzed whey-based formula (pHF-W) on growth and atopic dermatitis (AD) risk reduction in infants within the general infant population, and without a familial history of allergy as an inclusion or exclusion criterion. We reviewed the current evidence available from studies using pHF-W in the general population and summarized the data on safety (growth) and efficacy outcomes (reduction of AD), comparing the studies side by side. A total of 8 clinical trials were identified from the literature search, 7 of which used the same pHF-W. Six out of 8 studies indicated a reduction of atopic manifestations using a specific pHF-W versus cow's
\end{abstract}

\begin{tabular}{ll}
\hline KARGER & $\begin{array}{l}\text { (c) } 2018 \text { The Author(s) } \\
\text { Published by S. Karger AG, Basel }\end{array}$ \\
E-Mail karger@karger.com & This article is licensed under the Creative Commons Attribution- \\
www.karger.com/iaa & $\begin{array}{l}\text { NonCommercial-NoDerivatives 4.0 International License (CC BY- } \\
\text { NC-ND) (http://www.karger.com/Services/OpenAccessLicense). } \\
\text { Usage and distribution for commercial purposes as well as any dis- } \\
\text { tribution of modified material requires written permission. }\end{array}$
\end{tabular}

milk formula (CMF) in the first years of life. Data were summarized and compared side by side for growth (3 studies) and efficacy ( 5 studies). In these diverse general populations, the results on growth and AD were consistent with the previous findings reported on infants with a family history of allergy, but numerous limitations to these studies were identified. This literature review confirms that pHF-W supports normal growth in infants, and suggests that the risk of $A D$ may be reduced in not-fully breastfed infants from the general population when supplemented with a specific pHF-W when compared to CMF during the first 4-6 months of life. Further studies are warranted to confirm these results.

(C) 2018 The Author(s)

Published by S. Karger AG, Basel
Dr. Carine Blanchard

Institute of Nutritional Science, Nestlé Research Center Route du Jorat 57, PO Box 44

$\mathrm{CH}-1000$ Lausanne 26 (Switzerland)

E-Mail Carine.Blanchard@ rdyo.nestle.com 


\section{Introduction}

Allergy prevalence has been rising in the last few decades with atopic dermatitis (AD) being the most prevalent manifestation of allergy in infancy. AD affects $15-$ $30 \%$ of children; $60 \%$ of cases have an onset within the first year of life [1]. AD etiology is multifactorial, historically being associated with food allergy, and more specifically to milk allergy. AD etiology is now associated with genetic variants involved in skin barrier function defect and inflammation, leading/predisposed to a dry skin with increased susceptibility to environmental triggers [1]. While there are multiple specific etiologic factors for $\mathrm{AD}$, partially hydrolyzed whey-based formula (pHF-W) has been shown to be efficacious in reducing the risk of developing $\mathrm{AD}$ in the first years of life in infants with a first-degree relative affected by allergy [2-6]. Indeed, this population of infants is at a higher risk of developing allergy than the general population, and has been selected for numerous clinical trials to be sufficiently powered to demonstrate a significant effect of formula without recruiting massive numbers of infants. Hence, to date, the meta-analyses of pHF-W have mainly included studies on this high-risk population. However, about $50 \%$ of the infants who develop allergy do not fall in this category [7], and so it is not suspected and no prevention strategy is proposed.

During a workshop held in November 2014, we identified that the available information on the effects of pHF$\mathrm{W}$ in the general infant population was not accessible to the experts in the field, because it had not been published in English or had been presented at conferences but not published. We thus aimed at compiling all known available data and defining whether existing studies had determined whether $\mathrm{pHF}-\mathrm{W}$ can reduce the risk of developing $\mathrm{AD}$ in the general infant population. As recently suggested by the in-depth meta-analysis of Boyle et al. [8], pooling different studies performed with different products in the main analysis may hide the effect of specific infant formulas $[8,9]$.

In this study, we therefore identified and studied separately all types of clinical studies, prospective or retrospective, randomized or not, in which infants from the general population were enrolled, and without a familial history of allergy as an inclusion or exclusion criterion. In all of the included studies, infants were fed with either intact cow's milk formula (CMF) or pHF-W. The objective was to document and compare the studies; we thus summarized their results to assess the reproducibility of the findings on adequate growth, skin symptoms, $\mathrm{AD}$, and/or allergy onset. This review, due to the limitation of the data collected, is not intended to be a systematic review and meta-analysis. We thus solely present the results of this comparative summary, focusing on growth and AD risk reduction. Additionally, the strengths and limitations of the studies gathered are discussed in the light of their results.

\section{Methods}

Type of Studies

Randomized and non-randomized clinical trials, safety studies, observational studies, and epidemiologic studies with a prospective or retrospective design were included in the review. Specific study design was not an inclusion or exclusion criterion.

\section{Type of Participants}

Studies in which infants were selected from the general population (and for which a family history of allergy was neither an inclusion nor exclusion criterion) were eligible for inclusion.

\section{Type of Intervention}

We included any study in which infants from the general population (for which a family history of allergy was neither an inclusion nor exclusion criterion) were fed with $\mathrm{pHF}-\mathrm{W}$ versus an intact CMF who had had an intervention between birth and the age of 6 months.

\section{Type of Outcomes}

Studies including outcomes related to "allergy" or "atopy" or "allergic" or "atopic" or "dermatitis" or "eczema" or "infantile eczema" or "skin symptoms" or "sensitization" or "IgE" or "RAST" were eligible for inclusion.

\section{Search Methods}

The review was realized using available information obtained with the following research tools: PubMed, SCOPUS, and Google, and the studies previously identified in the meta-analyses, i.e., Boyle et al. [8], Alexander and Cabana [2], Osborn and Sinn [3], and Szajewska and Horvath [4]. Additionally, data were obtained from Nestlé Nutrition and the Nestlé Nutrition Institute, and this possibly led to a selection bias in this study. The authors of some of the studies were contacted to obtain the full poster presented at national or international congresses and any additional information.

\section{Assessment of the Risk of Bias}

The bias in the studies was assessed using methods similar to those recently published [6]. A low, medium, or high risk of bias was assigned to each study regarding selection, detection, performance, reporting, and other biases. When unknown, the risk was considered "medium".

\section{Summary and Representation of the Data}

The intention of the review is to present the studies side by side for similar outcomes without running a proper quantitative analysis or meta-analysis, as the design of the studies varied a lot and 
numerous risks for bias were identified. As such, data are shown individually using the same statistical parameters. All statistical analyses were performed using R v3.3.1. The proportion of allergic manifestations, eczema, or AD was compared between groups using Fisher's exact test. The conditional maximum likelihood estimate of the odds ratio (OR) and 95\% confidence interval (CI) were calculated. For allergy and skin manifestations, no adjustment for multiplicity was applied.

The mean weights were assumed normally distributed and compared between groups using $t$ tests. The mean differences are estimated and reported with a $95 \%$ CI. When multiple time points were reported, adjustment for multiplicity was performed, and $95 \%$ family-wise CI were reported for growth. To compute the $z$ scores for both sexes combined, for the trials conducted by Akimoto et al. [10, 11] and Wen et al. [17], these scores were calculated with the World Health Organization (WHO) tables for boys and girls separately, and then averaged. The mean differences in $z$ scores for both sexes combined are provided in Exl et al. [7].

\section{Dealing with Missing Data}

Intention to treat and per protocol analysis were not separated or mentioned in most of the studies. We thus show, when available, the ratio between the number of events and the total number of participants reported at the different time points, the OR for allergy-related parameters, and the $z$ scores (when available) for weight and length.

\section{Results}

\section{Study Selection}

The overview of the 8 studies assessed in this review is presented in Table 1, and study designs and details on excluded studies in the online supplementary material (see www.karger.com/doi/10.1159/000489861 for all online suppl. material). Out of these 8 studies, 7 used the same infant formula, a $100 \%$ pHF-W from Nestlé: Akimoto et al. [10, 11], Exl et al. [7, 12], Fukushima et al. [13], Hartman et al. [14], Ngamphaiboon [15], Schmitz et al. [16], and Wen et al. [17]. The eighth, performed by Moran [18], used a mix of pHF-W/casein (60/40) (Mead Johnson) and could not be summarized due to the lack of documentation on allergy-related parameters (only milkspecific IgG in the serum was reported); the authors also mentioned that the study was not powered to look at AD.

Among the remaining 7 studies performed with the same $100 \%$ pHF-W, 2 were not included in the reanalysis. The first, by Hartman et al. [14] did not provide sufficient quantitative data. The authors looked at growth between birth and the age of 6 months, and mentioned that mean growth velocity was similar across the different formulafed groups and did not significantly differ from the exclusively breastfed (BF) group over the 6-month period of the study. In addition, despite a significant reduction of

Effect of Partially Hydrolyzed Formula in the General Infant Population the rate of allergic manifestations for the pHF-W groups compared to the CMF group after 4 months of age, the study did not provide frequency data to allow for recalculating the effect [14]. The second, by Schmitz et al. [16], involved a very short intervention limited to the first 5 days of life, and did not provide sufficient numerical data for $\mathrm{AD}$.

Five of the 7 studies were reanalyzed for the risk of developing $\mathrm{AD}$, eczema, or allergic skin symptoms: Akimoto et al. [10,11], Exl et al. [7, 12], Fukushima et al. [13], Ngamphaiboon [15], and Wen et al. [17]. Only 3 studies provided sufficient data for growth assessment: Akimoto et al. [10, 11], Exl et al. [7, 12], and Wen et al. [17]. The study performed in Thailand by Ngamphaiboon [15] had to be excluded as it did not comment on growth, due to its retrospective design that could not guarantee scheduled visits. In addition, Fukushima et al. [13], while no differences in growth were observed between groups at 4 months, could not be included due to the lack of numerical data.

\section{Effect of pHF-W on Growth}

As discussed above, 3 of the 7 studies identified as using the same pHF-W consistently documented growth and safety data. The remaining 4 studies were excluded from the following summary.

\section{Published Results on Growth}

In Exl et al. [7, 12], health outcomes are recorded in detail and described. The authors mentioned that growth data (including weight, length, head circumference, and body mass index) were recorded by the physicians at scheduled visits according to standardized methods. Reference values were obtained from WHO standards available at that time $[19,20]$. No noticeable differences were observed in weight-for-age and length-for-age $z$ scores between the 2 cities from birth to 6 months, and adequate growth was observed in all groups compared to WHO standards $[19,20]$. The only significant confounder observed on growth was "smoking mother", associated with a lower weight (of $120 \mathrm{~g}$ at 3 months and $160 \mathrm{~g}$ at 6 months) compared to "non-smoking mother."

In Wen et al. [17], in which growth parameters (weight, length, and head circumference) at the ages of 1, 3, 6, 9, and 12 months were recorded, no statistical significant differences across the 3 groups were observed while using a global testing procedure. At 6 months, the weight was, on average, $0.2 \mathrm{~kg}$ higher in the CMF group compared to the $\mathrm{pHF}-\mathrm{W}$ group, i.e., $7.9 \pm 0.9 \mathrm{~kg}$ in the $\mathrm{pHF}-\mathrm{W}$ group, $8.1 \pm 0.9 \mathrm{~kg}$ in the CMF group, and $8.0 \pm 0.9 \mathrm{~kg}$ in the $\mathrm{BF}$ 


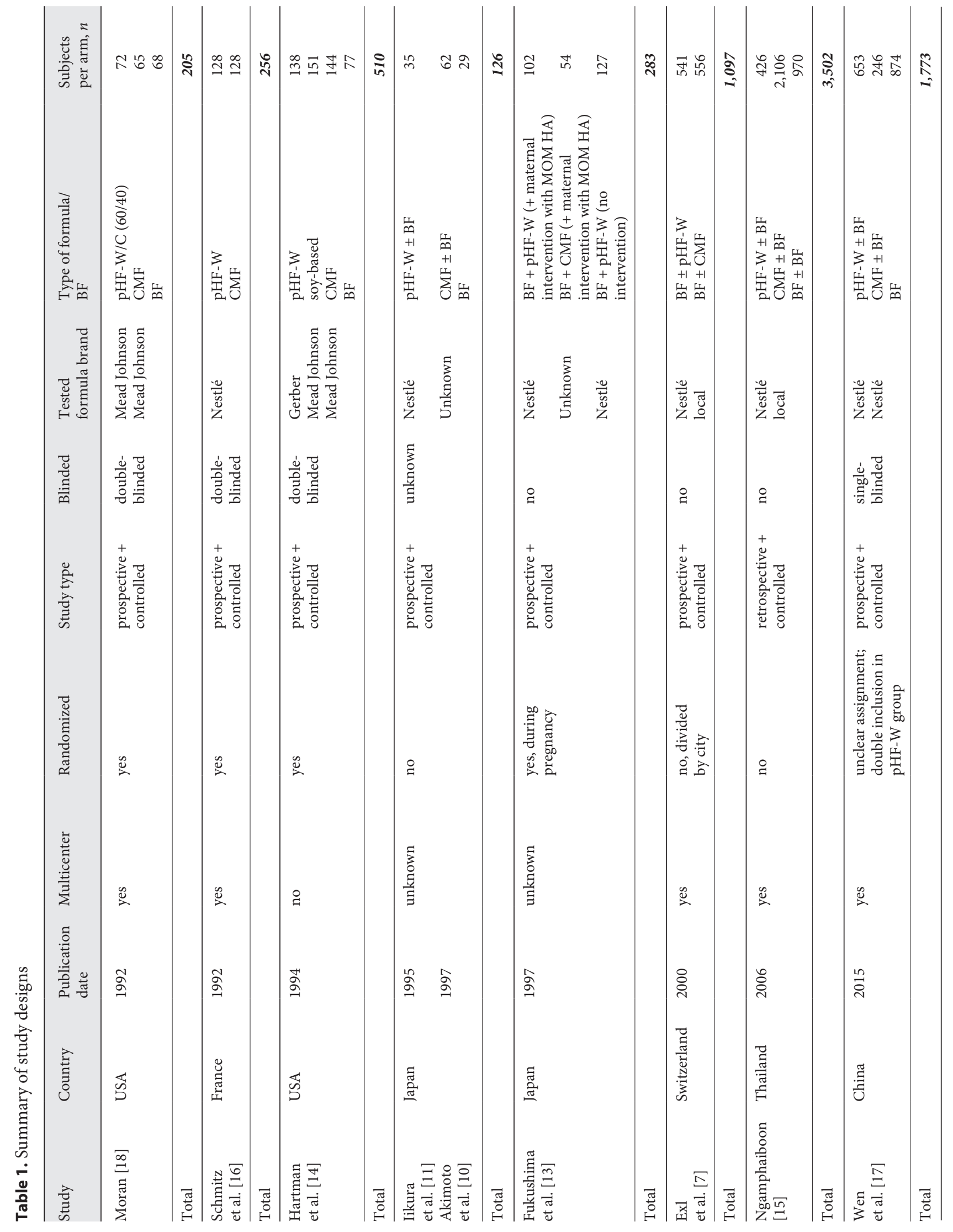




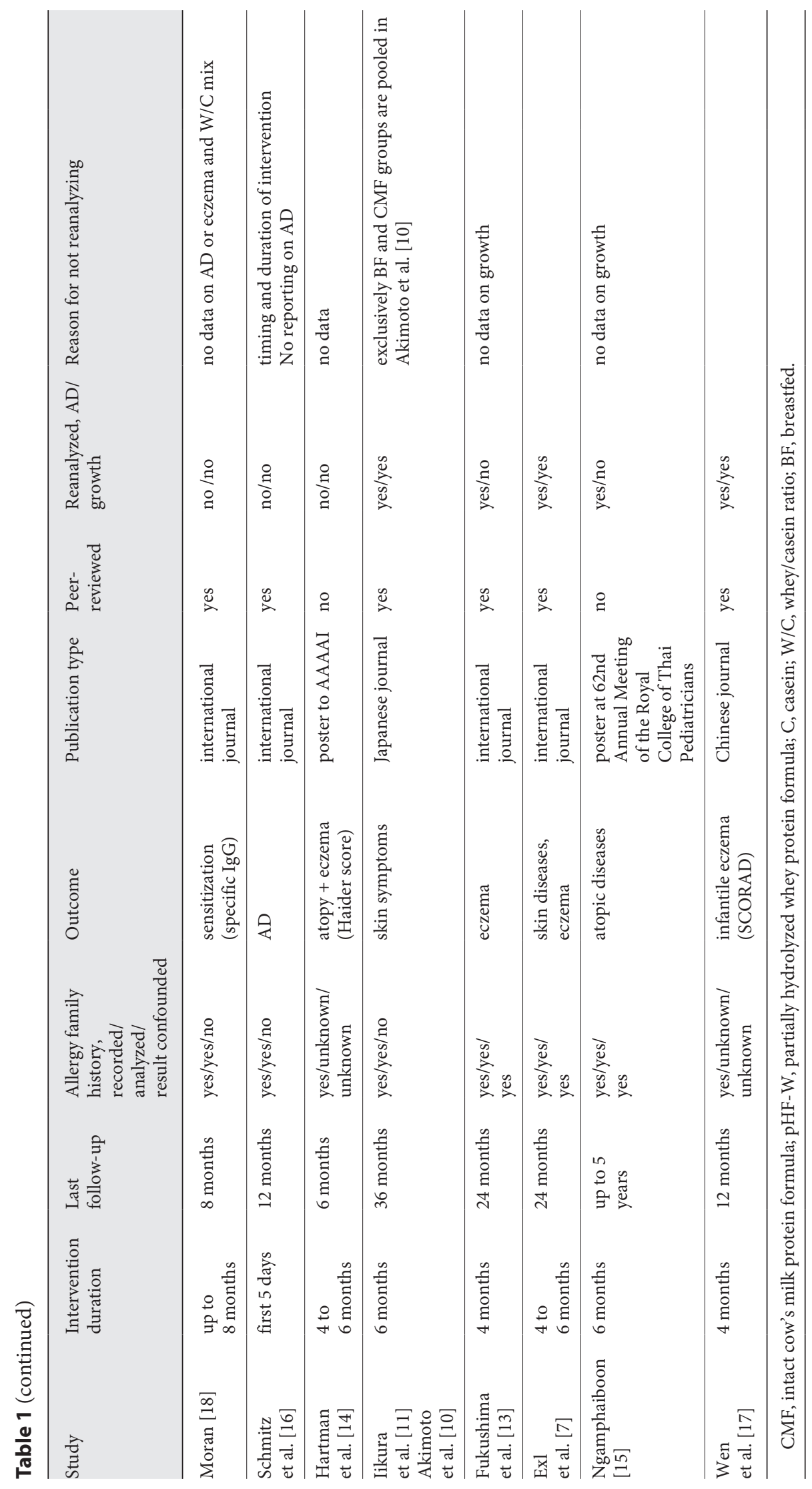


group. At 6 months, length was $67.2 \pm 2.5 \mathrm{~cm}$ in the pHF$\mathrm{W}$ group, $68.1 \pm 2.4 \mathrm{~cm}$ in the CMF group, and $67.6 \pm 2.3$ $\mathrm{cm}$ in the $\mathrm{BF}$ group.

Akimoto et al. [10] did not find any significant difference in body weight at 4 months between pHF-W and the infants fed either CMF or breast milk. Infants were 7,028.9 $\pm 729.4 \mathrm{~g}(n=35)$ in the pHF-W group versus 7,214.2 \pm $814.2 \mathrm{~g}(n=91)$ in the control CMF group combined with the exclusively BF group $(n=62+29$, respectively). Even though the average was $200 \mathrm{~g}$ more in the CMF group, differences were not significant, mostly due to the wide spread of the data observed in the compared groups. While the 2 groups, CMF and BF, were not combined in the original paper, the weights were not reported.

Summary of the Results

Figure 1 gives an overview of all the available results presented as $z$ scores compared to WHO standards or to a CMF group when individual data per group are not available. These data indicate that the growth (both weight and length) of the infants enrolled in these studies follows the WHO growth standard.

The differences between the 2 groups, i.e., $\mathrm{BF} \pm \mathrm{pHF}$ $\mathrm{W}(n=466)$ and $\mathrm{BF} \pm \mathrm{CMF}(n=535)$, for the weight-forage and length-for-age $z$ scores at 3 and 6 months from the study by Exl et al. [7] showed no difference between the 2 groups.

In Wen et al. [17], the growth measurements were done at 3, 6, 9, and 12 months of age. The weight results from the pHF-W group $(n=653)$ closely tracks the values observed for the BF group $(n=874)$. At every time point, the pHF-W group mean values are lower than the CMF group $(n=246)$, but within the WHO standard \pm 1 standard deviation (SD).

Finally, only the 4-month growth data were reported in the trial conducted by Akimoto et al. $[10,11]$ for the pHF-W $(n=35)$, CMF $(n=62)$, and BF $(n=29)$ groups. As shown in Figure 1, the mean weight-for-age $z$ score for the pHF-W group was lower than the CMF group, both being again within the range of the WHO standard.

\section{Effect of pHF-W on Allergy and AD Risk Reduction}

Five of the 7 studies using pHF-W were sufficiently documented for the allergic manifestations and/or $A D$ symptoms in the different intervention groups. In all studies, the family history of allergy was recorded. Most of them mentioned that the frequency of allergic parents and/or siblings was not significantly different in the CMF and pHF-W groups. However, 3 studies, Fukushima et al. [13], Ngamphaiboon [15], and Exl et al. [7], confounded their results with the increased risk of developing $\mathrm{AD}$ or other allergic manifestations. These 3 studies also mentioned that a family history of allergy was a main risk factor for atopic symptoms in the first year of life but the effect of the specific pHF-W was still seen after correcting for the allergy risk.

\section{Published Results on Allergic Symptoms Including}

AD

Based on the data reported in the study performed in Switzerland by Exl et al. [7], a significant decrease in AD incidence was observed in the pHF-W group at 6 months when the authors applied a standard statistical test $(11.9 \%$ in the non-intervention group vs. $7.0 \%$ in the intervention group).

In Wen et al. [17], the $\mathrm{AD}$ occurrences in the $\mathrm{BF}$ and pHF-W groups (27.00 vs. $27.26 \%)$ were similar. The incidence of $\mathrm{AD}$ in both the $\mathrm{BF}$ and $\mathrm{pHF}-\mathrm{W}$ groups was significantly lower than in the CMF group (34.96\%). These pairwise comparisons between groups were computed based on the data provided, and were not adjusted for multiple comparisons. This Chinese study suggests that a specific pHF-W reduces the risk of developing $\mathrm{AD}$ in the general infant population when compared to intact CMF. The results are also significant for the cumulative incidence of allergic manifestations, but are highly biased by the high prevalence of $\mathrm{AD}$ versus other allergic manifestations in this age group (Fig. 2).

In the trial reported in Japanese by Akimoto et al. [10], it is not clearly stated if the skin symptoms observed were related to $\mathrm{AD}$ or whether all types of skin symptoms were measured and included. However, in the English version of this study [11], the authors show the prevalence of allergic skin and respiratory symptoms at 4 and 12 months of age. A total of $3 / 35$ and 4/33 infants were affected by skin allergic symptoms in the pHF-W group at 4 and 12 months, respectively. At the same time points, 21/62 and $20 / 59$ infants were affected by skin allergic symptoms in the CMF group and 5/29 and 13/27 in the BF group, respectively. The authors observed a significant decrease of allergic symptoms (respiratory and skin allergic symptoms) in the pHF-W group at 4 months (OR 0.19; 95\% CI $0.03-0.70$ ) and 12 months (OR 0.27; 95\% CI 0.06-0.94) compared to the CMF group, and at 12 months compared to the BF group (OR 0.15; 95\% CI 0.03-0.61). Only $3 / 119$ subjects reported allergic respiratory symptoms at 12 months. In this study, the incidence of skin symptoms was followed up to 36 months of age, but was only significant at 1 year of age. The greatest difference was indeed observed at 1 year of age, with $5 / 33$ subjects (15.2\%) 


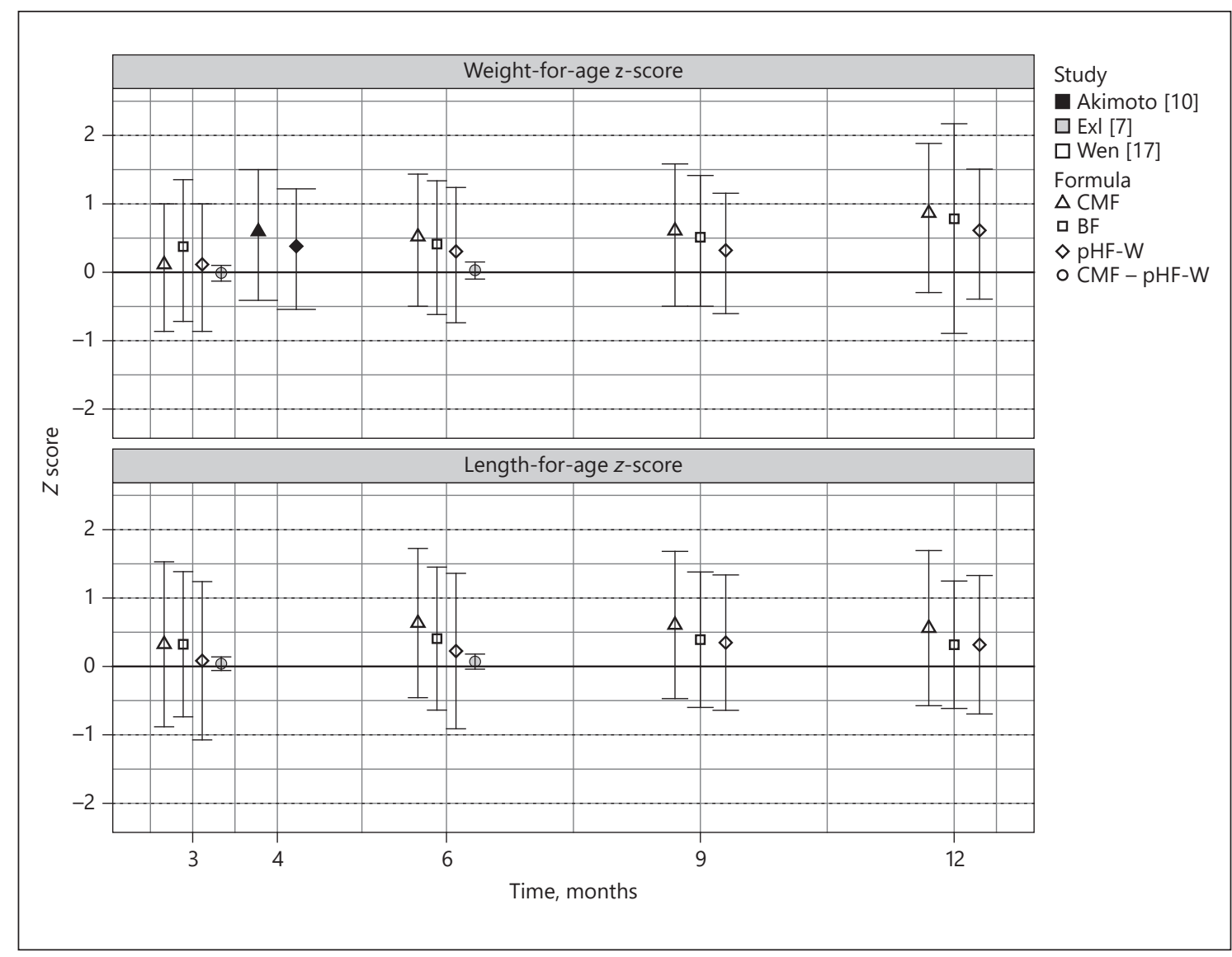

Fig. 1. Weight-for-age and length-for-age $z$ scores for both sexes combined. For Akimoto et al. [10] and Wen et al. [17], means and SD are represented for breastfed (BF) and formula-fed infants (CMF or pHF-W). For Exl et al. [7], the mean differences between pHF-W and CMF (i.e., CMF - pHF-W) with a 95\% confidence interval are shown, as only differential $\mathrm{z}$ scores were available.

presenting skin symptoms in the pHF-W group versus $37 / 86$ (43\%) in the CMF group (OR 0.24; 95\% CI 0.07$0.71)$.

In the study by Ngamphaiboon [15], the average age of the diagnosis of allergic diseases was 14.7 months (2 weeks to 5 years). The most prevalent clinical presentation in the first year of life was AD. The cumulative incidence of allergic manifestations was significantly reduced by using pHF-W compared with BF (OR 0.23 ; $95 \% \mathrm{CI}$ $0.160-0.314)$ and increased by using CMF compared with BF (OR 1.66; 95\% CI 1.396-1.957) when adjusted for sex and a family history of atopy. The direct comparison of the proportion of cumulative incidences between the CMF and pHF-W groups suggests that $30.3 \%$ of the $\mathrm{BF}$ group were atopic compared to $42.3 \%$ of the CMF group and $11.3 \%$ of the $\mathrm{pHF}-\mathrm{W}$ group. This was also significant when adjusting for sex and a family history of atopy.

Effect of Partially Hydrolyzed Formula in the General Infant Population
In the study by Fukushima et al. [13], at 4 months of age, the infants mixed-fed with breast milk and pHF-W showed a significantly lower prevalence of allergic manifestations and eczema than those fed breast milk complemented with CMF. No significant differences were found compared to the group that was fed exclusively breast milk. A significant decrease in eczema was found in the pHF-W group when compared to the CMF group at 12 months (OR 0.31; 95\% CI 0.13-0.75). The protective effect was not significant anymore at 24 months (OR 0.57; 95\% CI 0.24-1.37).

\section{Summary of the Study Results}

The results of study data are summarized in Figure 2 by comparing $\mathrm{AD}$, eczema, skin manifestations, and/or allergic manifestations between $\mathrm{pHF}-\mathrm{W}$ and CMF groups in the 5 selected studies. When possible, we focused on the cumu- 


\begin{tabular}{|c|c|c|c|c|c|c|}
\hline Study & pHF-W & CMF & OR $(95 \% \mathrm{Cl})$ & $p$ value & & \\
\hline Wen et al. (2015) - AD at 12 months & $178 / 653$ & $178 / 563$ & $0.70(0.50-0.97)$ & 0.027 & - & \\
\hline Wen et al. (2015) - atopic diseases at 12 months & $187 / 653$ & $91 / 246$ & $0.68(0.50-0.94)$ & 0.019 & $\square$ & \\
\hline Ngamphaiboon. (2006) - atopic diseases up to 5 years & $48 / 426$ & $891 / 2,106$ & $0.17(0.12-0.24)$ & $<0.001$ & - & \\
\hline Fukushima et al. (1997) - eczema at 12 months & & & $0.31(0.13-0.75)$ & $<0.01$ & $\square$ & \\
\hline Fukushima et al. (1997) - eczema at 24 months & & & $0.57(0.24-1.37)$ & ns & - & - \\
\hline Fukushima et al. (1997) - overall allergies at 6 months & & & $0.34(0.16-0.71)$ & $<0.05$ & $\square$ & \\
\hline Fukushima et al. (1997) - overall allergies at 24 months & & & $0.41(0.20-0.87)$ & $<0.05$ & 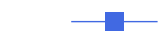 & \\
\hline Akimoto et al. (1997) - skin symptoms at 12 months & $5 / 33$ & $37 / 86$ & $0.24(0.07-0.71)$ & 0.005 & $\square$ & \\
\hline likura et al. (1995) - skin symptoms at 4 months & $3 / 35$ & $21 / 62$ & $0.19(0.03-0.70)$ & 0.006 & 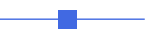 & \\
\hline \multirow[t]{2}{*}{ likura et al. (1995) - skin symptoms at 12 months } & $4 / 33$ & $20 / 59$ & $0.27(0.06-0.94)$ & 0.027 & + & \\
\hline & & & & & $\begin{array}{rr}0.1 \quad 0.250 .5 \\
\\
\quad \text { OR }\end{array}$ & 1 \\
\hline
\end{tabular}

Fig. 2. Effect of infant feeding on the cumulative incidence of eczema, skin symptoms, atopic diseases, or AD: differential effects of pHF-W versus CMF. For Fukushima et al. [13], only odds ratios (ORs) with 95\% confidence intervals (CIs) of prevalence are reported.

lative incidence of $\mathrm{AD}$ or eczema $[7,15,17]$, but all available data are presented. Our data were based on the data reported in the papers but were not adjusted for confounders such as a family history of allergy or multiple testing.

The Wen et al. [17] and Ngamphaiboon [15] studies mentioned that $\mathrm{AD}$ was the main allergic symptom recorded in the first years of life. Approximately $95 \%$ of the recorded allergy cumulative incidence in Wen et al. [17] consisted of $\mathrm{AD}$ manifestations; this study involves $\mathrm{AD}$ and atopic diseases whereas Ngamphaiboon [15] covered atopic disease only.

In Akimoto et al. [10, 11], the effect of pHF-W versus CMF on skin symptoms is shown. However, in Fukushima et al. [13], while eczema and overall allergies are reported, only prevalence ORs are reported in the forest plot (Fig. 2) because the exact prevalence ratio was not provided in the related publication.

When comparing pHF-W to CMF (Fig. 2), the ORs are in favor of the pHF-W for allergic manifestations (Wen et al. [17]: OR 0.68; 95\% CI 0.50-0.94, $p=0.019$; Ngamphaiboon [15]: OR 0.17; 95\% CI 0.12-0.24, $p<0.001)$ and $\mathrm{AD}$ (Wen et al. [17]: OR 0.70; 95\% CI 0.50-0.97, $p=0.027$; Exl et al. [7]: OR 0.56; 95\% CI 0.36-0.87, $p=0.007)$. Fukushima et al. [13] and Akimoto/Iikura et al. [10/11] also show a significant effect of pHF-W on allergy or eczema, mostly in the first year of life (Fig. 2). No significant reduction of the risk of developing eczema at 24 months and of developing overall allergies at 18 months were observed in Fukushima et al. [13]. Comparison of the pHF$\mathrm{W}$ or CMF group to the BF group was also performed; results are described in the supplementary results and shown in online supplementary Figure 1.

\section{Study Bias and Limitations}

Figure 3 and Table 1 summarize the bias identified in these studies. The selection of the participants was not randomized in some of the studies, as shown in Table 1. In some studies, the mothers were free to choose their 


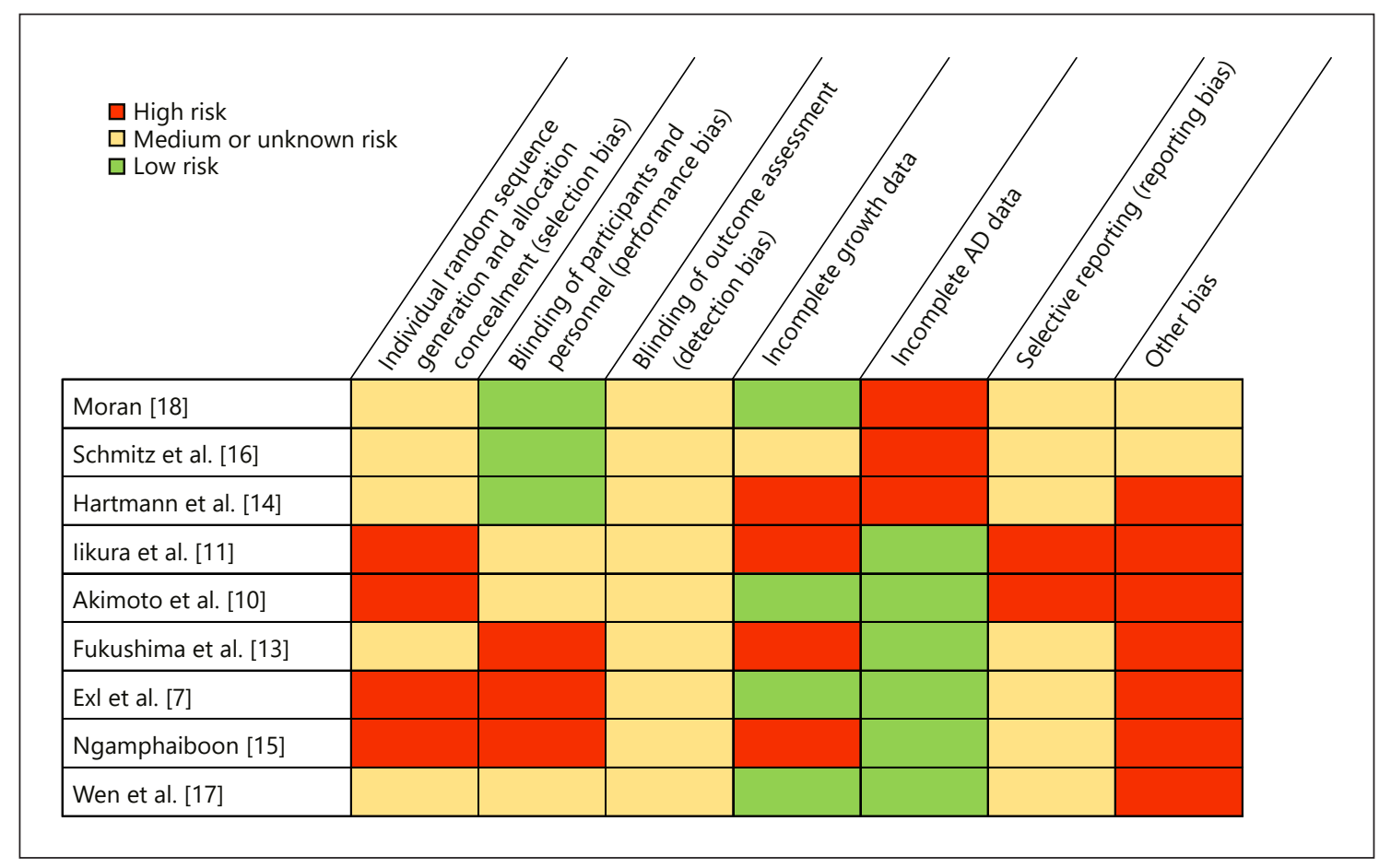

Fig. 3. Limitations and biases of the studies. The studies were assessed for selection, detection, performance attrition, reporting, and other biases. A color code was used. Green, low risk; orange, medium or unknown risk; red, high risk.

preferred formula type according to study protocol guidelines $[10,11,15,17]$. When randomized, the use of random sequence generation or allocation concealment is not described, suggesting a medium-to-high risk of selection bias in these studies overall. Only 3 studies [14, 16, 18] were clearly mentioned as double-blinded, but in these, the data on growth or $\mathrm{AD}$ were not available from the publication. One study was single-blinded [17], one was a retrospective observational study [15], and the others did not report on the blinding of the participants or health care practitioners. Similarly, in all of the studies, the blinding of the outcome assessment/the method of data collection was not reported in the publication, suggesting a medium-to-high risk of a performance and detection bias. The type of outcome reported is also not consistent across studies. Allergy, AD, and eczema are frequently used interchangeably but they are different entities. Allergy regroups symptoms that are linked to an adverse reaction to an environmental or food allergen that is innocuous to non-allergic individuals. The symptoms of allergy can affect different organs such as the lungs, gastrointestinal tract, and skin, or even lead to cardiovascular manifestations in the case of anaphylaxis. AD is a chronic inflammation of the skin characterized by patches of relapsing itchy rashes and lesions. As mentioned in the Introduction, $\mathrm{AD}$ etiology is multifactorial and is one of the manifestations of food allergy [1]. Eczema is an entity that regroups numerous skin manifestations, including, but not limited to, AD. The limited information regarding the exact definition of the investigated outcomes in some of the studies referred in this review is also to be considered as a possible bias.

It is possible that there was selective reporting in all of the studies, and this is clearly observed when comparing the publications by Akimoto et al. [10] and likura et al. [11] on the same trial. The selective reporting of positive results certainly led to a bias in this review as non-positive findings are frequently not reported in publications, mostly due to the low interest of readers and journals for negative data. When present, negative data are only cited as non-significant, limiting access to the numerical data. However, out of the studies that fall in the category of data not being reported, Moran [18] and Schmitz et al. [16] did not look at the outcome due to not being powered enough or not finding any significant effect, while Hartman et al. [14] mentioned a significant protective effect of the pHF-W over the CMF. 
As far as other biases are concerned, such as concomitant nutritional recommendations other than the tested formula, the absence of peer-reviewed publication in an international journal, or the sponsorship provided by the company manufacturing the formula, all but 2 of the studies were identified as having a higher risk of bias.

Additionally, while all identified studies using pHF-W could be included in this review, a selection bias exists due to the access and information obtained from one-manufacturer internal data. Altogether, these analyses suggest that there is interesting data on growth and AD risk reduction with PHF-W supplementation in the general infant population, but these data need to be interpreted in the light of the above limitations.

\section{Discussion}

Breastfeeding is the ideal feeding option for infants. The introduction of formula as a breast milk substitute should only be suggested if the mother cannot or does not want to breastfeed. Currently, pHF-W is approved by the European Food Safety Authority (EFSA) and the US Food and Drug Administration (FDA) for use as a routine infant formula for any healthy term infant.

In this review, we aimed at compiling the evidence regarding growth and allergic manifestations in infants from the general population (for which a family history of allergy was neither an inclusion nor an exclusion criterion) who were supplemented with $\mathrm{pHF}-\mathrm{W}$ during the first 4-6 months of life.

We identified 8 studies fitting our selection criteria, regrouping a total of 7,752 infants, 2,222 of which were fed pHF-W or pHF-W/casein (60/40). Of these 8, 6 demonstrated a significant decrease of $\mathrm{AD}$ or allergic/skin manifestations with pHF-W (Akimoto et al. [10, 11], Exl et al. [7, 12], Fukushima et al. [13], Hartman et al. [14], Ngamphaiboon [15], and Wen et al. [17]). Five displayed sufficient data on allergy symptoms to be reported, totaling 1,757 infants fed with the same pHF-W (Akimoto et al. [10, 11], Exl et al. [7, 12], Fukushima et al. [13], Ngamphaiboon [15], and Wen et al. [17]). Regarding growth, 3 of these 5 allowed us to reanalyze the effect of this specific pHF-W on infant growth by incorporating data on 1,154 infants (Akimoto et al. [10,11], Exl et al. [7, 12], and Wen et al. [17]). The studies show weights in the pHF-W group to be similar to the $\mathrm{BF}$ group and in the range of WHO standards. While a trend toward a $200-\mathrm{g}$ increase in the CMF group (compared to the pHF-W group) was seen in 2 studies, suggesting a trend toward slight over- nutrition after the age of 4 months, the averages are within the normal range the SDs are quite large, and complementary foods are usually already introduced. A larger study would be needed to assess the clinical relevance of this observation about CMF.

A meta-analysis at 12 months that included the results from Fukushima et al. [13], Wen et al. [17], and Akimoto et al. [10] was performed on eczema, $\mathrm{AD}$, and allergic skin symptoms, respectively, and showed a significant protective effect in favor of pHF-W compared to CMF (online suppl. Fig. 2). Indeed, the effect size (a $30-40 \%$ risk reduction at 1 year of age) is comparable to what has been described in a meta-analysis of infants with a family history of $\mathrm{AD}[2-4,6]$. However, due to the variability of study design, the different allergic outcomes, the various infant ages at which allergic outcomes were measured, and the possible study selection bias identified in this review, the relevance of the meta-analysis remains low. Indeed, the criteria for the identification of atopy, allergic manifestations, or AD differed, and was not clearly or uniformly described in all studies. For this reason, individual study results were reported rather than performing a metaanalysis. In addition, the US and European agencies (US FDA and EFSA) usually perform their scientific assessment for clinical substantiation on multiple individual studies using the same product and clinical outcomes rather than performing meta-analyses.

A first interesting observation of this analysis relates to the geographic distribution of the included studies: 1 in Europe [7], 1 in China [17], 1 in South East Asia [15], 1 in the USA [14], and 2 in Japan $[10,11,13]$. This indicates the wide geographical interest in reducing the risk of developing atopic diseases by nutritional intervention. It also suggests that ethnic differences between populations and environmental components may not necessarily compromise the preventive effect of pHF-W on $\mathrm{AD}$ or allergic manifestations in the first year of life.

This multiple individual study report is subject to some limitations inherent to the design of the studies. As always, the $\mathrm{BF}$ reference groups were not randomized. In some studies, breastfeeding was exclusive, while mixed feeding was present in some others. Of note, in 2 studies [7, 10], the authors combine exclusively BF infants, formula-fed infants, and partially BF infants in the CMF and pHF-W groups. In Akimoto et al. [10], the CMF and BF groups were combined in a single control group, thus rendering the interpretation more difficult. It is also highly likely that mothers switched formula in the retrospective study performed in Thailand [15]. In this study, the requirement for feeding exclusively with 1 type of formula is not reported. 
Interestingly, the effect size observed with pHF-W supplementation is similar to what was observed in trials performed in populations selected with a first-degree relative with allergic disease [2-6]. On explanation could be that, in the general infant population with the genetic bias being weaker than in infants with a first-degree relative with allergic disease, the effect of a change like pHF-W versus CMF may have a better or similar effect due to being less confounded by genetic factors that would favor the development of allergic symptoms regardless of nutritional or environmental pressures. On the other hand, this could have been due to the unequal distribution of allergy risk in the different groups. A subgroup analysis of these studies, separating infants according to risk, may help answer whether the effect of pHF-W is similar in infants with and without $\geq 1$ first-degree relatives presenting allergy. While the family history of allergy was recorded, subgroup analyses were mostly not performed, and results were confounded for allergy risk in 3 of the studies $[7,13,15]$. However, these studies did report a significant reduction of the risk of developing $\mathrm{AD}$ even once the data were confounded with the family allergy risk. This type of analysis is particularly important for non-randomized trials and/or when the mothers were made aware of the benefit of pHF-W on allergy risk reduction as in Wen et al. [17]. In non-randomized studies, there is also a high risk that mothers breastfeed for longer or give pHF-W if symptoms start while they are still fully breastfeeding. This may explain, in part, the effect observed in the nonrandomized retrospective trial performed by Ngamphaiboon [15].

Specific late introduction of complementary allergenic food was recommended in Exl et al. [7]. In this study, the authors measured the effect of a dietary recommendation on $\mathrm{AD}$ risk reduction as they combined a recommendation to introduce pHF-W feeding when exclusive breastfeeding was not possible with a dietary recommendation for introducing complementary foods [8]. This recommendation of late introduction of highly allergenic food only in the pHF-W group may have partly influenced AD incidence.

Delaying solid food introduction including allergenic foods after the age of 1 year is no longer recommended for preventing allergic diseases [21]. Other environmental factors may influence the incidence of allergic manifestations as local habits may modify allergic susceptibility even if cities are close to each other, like in the study by Exl et al. [7]. Indeed, von Mutius et al. [22-24] have shown that, even in the same village, early life exposure to dairy farms influences the prevalence of asthma and allergic rhinitis in children, thereby highlighting the necessity for proper randomization. Most of the studies did not report on compliance, and the dropout rate (with/ without reason) was not always mentioned. Intention-totreat (ITT) and per-protocol (PP) analyses were discussed in the Exl et al. [7] study only. Finally, there was not the same amount of data available in the different trials and 2 of the works were never peer-reviewed and only submitted as abstracts. Altogether, and knowing these limitations, this multiple individual study analysis ascribes value to this existing data and provides additional relevant results for growth, allergy risk reduction, and $\mathrm{AD}$ in the general infant population.

In conclusion, the available studies suggest that, when breastfeeding is not possible, feeding a specific pHF-W instead of intact CMF may reduce the risk of developing atopic manifestations, including $\mathrm{AD}$, in the general infant population, while still supporting age-appropriate infant growth. A well-designed, randomized, double-blinded study or an independent, single-blinded study comparing supplementation with pHF-W versus CMF is warranted to confirm these results.

\section{Acknowledgments}

This work originated from a workshop held in November 2014. The authors wish to thank Yvonne Vissers, $\mathrm{PhD}$, for organizing the workshop, Valerie Verhasselt $\mathrm{MD}, \mathrm{PhD}$, for her participation in the workshop, and Marco Turini MD and Jose Saavedra, MDs, for reviewing the manuscript. The authors are grateful to the families participating in these studies and the clinical investigators involved in the 8 identified studies.

\section{Statement of Ethics}

The authors have no ethical conflicts to disclose.

\section{Disclosure Statement}

This manuscript is an outcome of workshop discussions organized in November 2014 by the Nestlé Research Center to which N.G., C.B, J.M.S., H.U.S., D.S., and S.K. participated. J.S., S.N., S.P., N.G., and C.B, are employed by Nestec Ltd. S.K. is the recipient of grants and research supports from BioGaia, Nestlé Nutrition, Mead Johnson, and R-Biopharm, received consultation fees from Biocodex, Danone, Nestle Nutrition, Boehringer Ingelheim, Shire, Janssen, and Abbvie, and participated in company-sponsored speaker's bureaus with Nestlé Nutrition, ThermoFisher, Mead Johnson, Danone, Abbvie, and Shire. H.U.S., D.S., and J.M.S. have no conflict of interest to disclose in the context of this work. 


\section{Funding Sources}

This manuscript is an outcome of workshop discussions organized and financed in November 2014 by the Nestlé Research Center.

\section{Author Contributions}

C.B, N.G., J.M.S., D.S., H.U.S., and S.K. participated in the ideation of the manuscript; C.B and J.S. reviewed the literature and analyzed the data, C.B and S.N. drafted the manuscript, all the authors constructively reviewed and agreed with the manuscript.

\section{References}

1 Bieber T: Atopic dermatitis. N Engl J Med 2008;358:1483-1494.

2 Alexander DD, Cabana MD: Partially hydrolyzed $100 \%$ whey protein infant formula and reduced risk of atopic dermatitis: a meta-analysis. J Pediatr Gastroenterol Nutr 2010;50: 422-430.

3 Osborn DA, Sinn J: Formulas containing hydrolysed protein for prevention of allergy and food intolerance in infants. Cochrane Database Syst Rev 2006;CD003664.

4 Szajewska H, Horvath A: Meta-analysis of the evidence for a partially hydrolyzed 100\% whey formula for the prevention of allergic diseases. Curr Med Res Opin 2010;26:423437.

5 von Berg A, Koletzko S, Grubl A, Filipiak-Pittroff B, Wichmann HE, Bauer CP, et al: The effect of hydrolyzed cow's milk formula for allergy prevention in the first year of life: the German Infant Nutritional Intervention Study, a randomized double-blind trial. J Allergy Clin Immunol 2003;111:533-540.

6 Hania Szajewska H, Horvath A: A partially hydrolyzed $100 \%$ whey formula and the risk of eczema and any allergy: an updated metaanalysis. World Allergy Organ J 2017;10:27.

7 Exl BM, Deland U, Secretin MC, Preysch U, Wall M, Shmerling DH: Improved general health status in an unselected infant population following an allergen-reduced dietary intervention programme: the ZUFF-STUDYPROGRAMME. Part II: infant growth and health status to age 6 months. ZUg-FrauenFeld. Eur J Nut 2000;39:145-156.

8 Boyle RJ, Ierodiakonou D, Khan T, Chivinge J, Robinson Z, Geoghegan N, et al: Hydrolysed formula and risk of allergic or autoimmune disease: systematic review and metaanalysis. BMJ 2016;352:i974.
9 Koletzko S, Filipiak-Pittroff B, Heinrich J, Koletzko B, Berdel D, von Berg A: Hydrolysed formula and risk of allergic or autoimmune disease: systematic review and meta-analysis. BMJ 2016;352:i974.

10 Akimoto K, Saito H, Akasawa A, Iikura Y: Preventative effect of a whey hydrolyzed formula [Nestle, NAN H.A.] on the development of allergic symptoms in infants. Arerugi 1997; 46:1044-1051.

11 Iikura Y, Akimoto K, Ebisawa M, Onda T, Akasawa A, Saito H, et al: Effect of hydrolyzed whey protein formula for babies on development of allergic symptoms during infancy. Nestle Nutr Workshop Ser Pediatr Program 2016;34:269-275.

12 Exl BM, Deland U, Secretin MC, Preysch U, Wall $\mathrm{M}$, Shmerling DH: Improved general health status in an unselected infant population following an allergen reduced dietary intervention programme. The ZUFF-studyprogramme. Part I: study design and 6-month nutritional behaviour. Eur J Nutr 2000;39: 89-102.

13 Fukushima Y, Iwamoto K, Takeuchi-Nakashima A, Akamatsu N, Fujino-Numata N, Yoshikoshi M, et al: Preventive effect of whey hydrolysate formulas for mothers and infants against allergy development in infants for the first 2 years. J Nutr Sci Vitaminol 1997;43: 397-411.

14 Hartman C, Fredericks G, Katz E, Brown C: Prevalence of symptomatic formula intolerance and allergy in a general infant population. J Allergy Clin Immunol 1994;93:210
15 Ngamphaiboon J: Only you can prevent childhood allergy. J Med Assoc Thai 2006: 89:S169-S170.

16 Schmitz J, Digeon B, Chastang C, Dupouy D, Leroux B, Robillard P, et al: Effects of brief early exposure to partially hydrolyzed and whole cow milk proteins. J Pediatr 1992;121(5 Pt 2):S85-S89.

17 Wen S, Zhijian W, Mei Z, Qiao X, Yucong C, Mailian D, et al: Preventive effect of prenatal education and partially hydrolyzed whey formula on infantile allergy: a prospective study. Chin J Perinat Med 2015;18:904-909.

18 Moran JR: Effects of prolonged exposure to partially hydrolyzed milk protein. J Pediatr 1992;121(5 Pt 2):S90-S94.

19 WHO Working Group on Infant Growth: An Evaluation of Infant Growth. Geneva, World Health Organization, 1994.

20 WHO Working Group on Infant Growth. An evaluation of infant growth: the use and interpretation of anthropometry in infants. Bull World Health Organ 1995;73:165-174

21 Fleischer DM, Spergel JM, Assa'ad AH, Pongracic JA: Primary prevention of allergic disease through nutritional interventions. J Allergy Clin Immunol Pract 2013;1:29-36.

22 von Mutius E, Braun-Fahrlander C, Schierl R, Riedler J, Ehlermann S, Maisch S, et al: Exposure to endotoxin or other bacterial components might protect against the development of atopy. Clin Exp Allergy 2000;30:12301234.

23 Riedler J, Braun-Fahrlander C, Eder W, Schreuer M, Waser M, Maisch S, et al: Exposure to farming in early life and development of asthma and allergy: a cross-sectional survey. Lancet 2001;358:1129-1133.

24 Von Ehrenstein OS, von Mutius E, Illi S, Baumann L, Böhm O, von Kries R: Reduced risk of hay fever and asthma among children of farmers. Clin Exp Allergy 2000;30:187-193. 